\title{
Visualizing sporadic E using aeronautical navigation signals at VHF frequencies
}

\author{
Keisuke Hosokawa ${ }^{1, *}$, Kotaku Kimura ${ }^{1}$, Jun Sakai ${ }^{1}$, Susumu Saito ${ }^{2}$, Ichiro Tomizawa ${ }^{3}$, \\ Michi Nishioka ${ }^{4}$, Takuya Tsugawa ${ }^{4}$, and Mamoru Ishii ${ }^{4}$ \\ ${ }^{1}$ Department of Communication Engineering and Informatics, University of Electro-Communications, Chofugaoka 1-5-1, \\ Chofu, 182-8585 Tokyo, Japan \\ ${ }^{2}$ Electronic Navigation Research Institute, National Institute of Maritime, Port and Aviation Technology, Jindaiji-Higashicho 7-42-23, \\ Chofu, 182-0012 Tokyo, Japan \\ 3 Center for Space Science and Radio Engineering, University of Electro-Communications, Chofugaoka 1-5-1, Chofu, 182-8585 Tokyo, Japan \\ ${ }^{4}$ National Institute of Information and Communications Technology, Nukui-Kitamachi 4-2-1, Koganei, 184-8795 Tokyo, Japan
}

Received 31 July 2020 / Accepted 6 December 2020

\begin{abstract}
To understand the dynamical properties of sporadic E layer (Es), we have developed a method to visualize the two-dimensional spatial structure of Es by monitoring the occurrence of anomalous propagation of VHF waves used for aeronautical navigation systems in combination with the electron density disturbance index ROTI, that is the Rate of total electron content (TEC) Index obtained from ground-based GPS receivers. We introduce a case of strong Es layer which occurred for $\sim 4 \mathrm{~h}$ during daytime of July 4, 2019. In this interval, we succeeded in imaging the structure of Es elongating more in the east-west direction and moving northward gradually. This result indicates that the combination of network observations of aeronautical navigation signals and ROTI is capable of imaging the 2D structure of Es in a wide area including the sea surface, which enables us to discuss the dynamical characteristics and generation mechanism of Es.
\end{abstract}

Keywords: mid-latitude ionosphere / sporadic E / VHF radio observations / GPS-TEC measurements

\section{Introduction}

The sporadic E layer (Es) has been known as one of the outstanding phenomena in the E region ionosphere. During intervals of Es, the electron density at an altitude of $100 \mathrm{~km}$ is extremely enhanced, often exceeding the maximum electron density in the F region (Whitehead, 1989; Mathews, 1998; Haldoupis, 2011). At mid-latitudes, Es generally appears in summer months with two occurrence peaks in the daytime and nighttime, respectively (i.e., semi-diurnal variation in its local time distribution). An increase of the critical frequency due to Es has a potential to cause reflection of radio waves of oblique incidence at frequencies up to $150 \mathrm{MHz}$ (Davis et al., 1959). The effects of Es on analogue television and FM radio in such a frequency range have been well-known for a long time. Recently, Sakai et al. (2019, 2020) demonstrated that propagation of VHF radio waves used for aeronautical navigation (so-called NAV signals at $108-118 \mathrm{MHz}$ range) has also been affected by the appearance of Es. In particular, they statistically showed that Es anomalous propagation (EsAP) of NAV

\footnotetext{
*Corresponding author: keisuke.hosokawa@uec. ac. jp
}

signals often occurs in Japan during summer months. Following these studies, routine monitoring of such EsAP cases has started at six stations in Japan since 2019 (Hosokawa et al., 2020), which can be used for evaluating the influence of Es in a wide area.

Because of its effects on radio communications, Es has still been actively investigated in the recent decades by using various remote sensing techniques and numerical modeling. It has been suggested that Es is produced by a concentration of metallic ions by a vertical shear in the neutral wind (e.g., Whitehead, 1989). Recently, Shinagawa et al. (2017) succeeded in explaining the seasonal and local time dependences of Es by considering the wind shear theory as a generation mechanism of Es. However, the spatial structure and dynamical characteristics of Es have not yet been revealed in detail. Thus, two-dimensional visualization and dynamical tracking of Es are highly demanded.

There are several existing methods for observing Es in 2D fashion, for example, the one using VHF radar imaging technique (Hysell \& Burcham, 2000; Saito et al., 2006). This approach is good at capturing small-scale structures associated with Es, but the application of this method is limited only for 
nighttime Es accompanied by meter-scale plasma irregularities. Another method for the 2D observation of Es is the use of GPS-TEC (total electron content) measurements. Maeda \& Heki (2015) visualized the two-dimensional spatial structure of Es by mapping the deviation component of TEC (delta TEC) to $100 \mathrm{~km}$ altitude (i.e., the altitude of Es). By mapping the 2D structure of 71 Es events in 2010, they statistically showed that Es has an east-west elongating structure in most cases and often moves in a direction perpendicular to the direction of elongation (i.e., mostly meridional). They also indicated that Es has a migration velocity of $30-100 \mathrm{~m} \mathrm{~s}^{-1}$, average value being about $60 \mathrm{~m} \mathrm{~s}^{-1}$, and the spatial extent is $50-500 \mathrm{~km}$ in east-west and $10-30 \mathrm{~km}$ in north-south direction, respectively. In addition to GPS-TEC, InSAR technique has also been used to detect the fine-scale structure of Es (Maeda et al., 2016; Furuya et al., 2017).

In this paper, we introduce a 2D mapping method of Es that combines the observation of EsAP of VHF NAV signals with the rate of TEC index (ROTI) from ground-based GPS receivers. This combined approach enables us to visualize the spatial extent and dynamical characteristics of Es in a wide area.

\section{Method}

To monitor the occurrence of EsAP in NAV band, that are VOR (VHF omnidirectional radio range) and ILS LOC (Instrument Landing System Localizer), we have been operating receivers at six locations in Japan: Sarobetsu $\left(45.16^{\circ} \mathrm{N}\right.$, $\left.141.75^{\circ} \mathrm{E}\right)$, Oarai $\left(36.33^{\circ} \mathrm{N}, 140.58^{\circ} \mathrm{E}\right)$, Chofu $\left(35.66^{\circ} \mathrm{N}\right.$, $139.54^{\circ}$ E), Sugadaira $\left(36.42^{\circ} \mathrm{N}, 138.32^{\circ} \mathrm{E}\right)$, Kure $\left(34.24^{\circ} \mathrm{N}, 132.53^{\circ} \mathrm{E}\right)$ and Onna $\left(26.50^{\circ} \mathrm{N}, 127.85^{\circ} \mathrm{E}\right)$ since May 2019 (Hosokawa et al., 2020). As shown in Figure 1, these receiving $(\mathrm{Rx})$ stations are aligned along the Japan islands extending from southwest to northeast. At each station, we continuously receive radio waves in the NAV band from 108 to $118 \mathrm{MHz}$. The source transmitting (Tx) stations of NAV signals are indicated by the black dots in Figure 1, which are distributed mainly in East Asia.

The spectrum analysis is performed for the received raw waveform onsite and the signal strength is recorded for each of 193 NAV channels with a temporal resolution of $10 \mathrm{~s}$. The data include information on the time of signal reception, frequency, receiving station and received signal intensity. As will be described later in detail, we map the spatial distribution of Es by considering the mid-points between all the Tx and Rx stations as locations where the NAV signals are reflected by Es. The distribution of such mid-points is shown in Figure 1 by the cross marks whose color represents the corresponding $\mathrm{Rx}$ station. The cluster of these cross marks indicates the coverage (or field-of-view) of the Es imaging using EsAP.

The mapping of Es using EsAP should be complemented with other mapping methods since the field-of-view of the measurement depends on the location of $\mathrm{Tx}$ and $\mathrm{Rx}$ stations. Therefore, we make use of the rate of TEC index (ROTI: Pi et al., 1997), which is the standard deviation of the $5 \mathrm{~min}$ variability of GPS-TEC, for detecting electron density irregularities embedded within Es. ROTI can be derived by the following equation $(\mathrm{ROT}=$ time difference of TEC in $30 \mathrm{~s}, \delta t=30 \mathrm{~s}$ in the following equation):

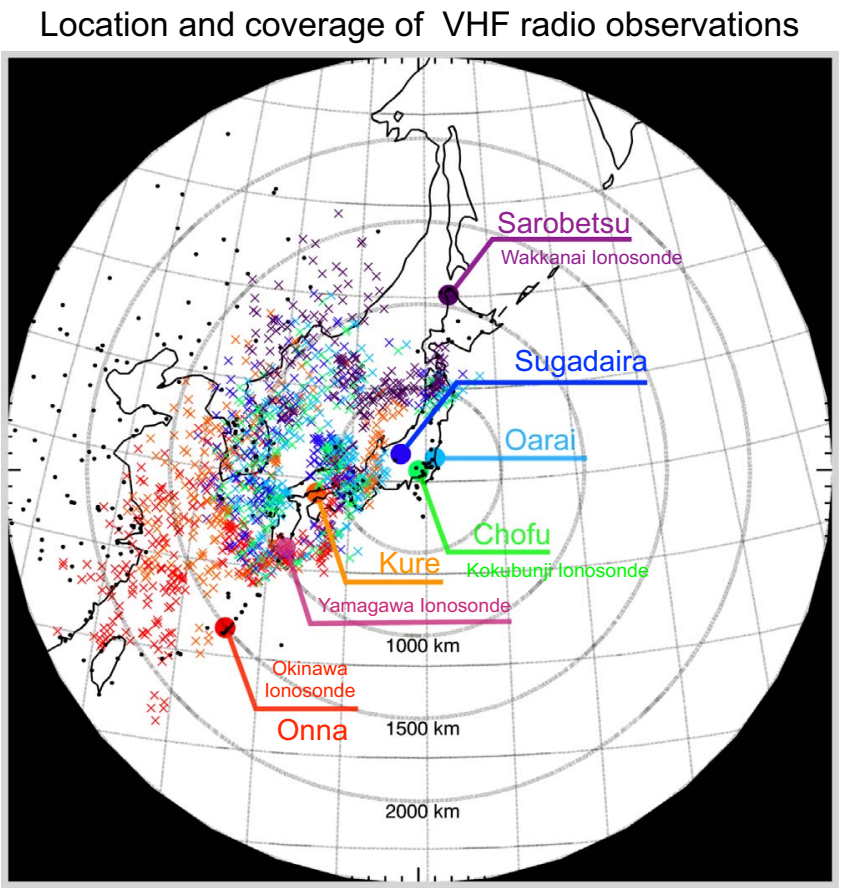

Fig. 1. Map of six Rx stations (Sarobetsu, Sugadaira, Oarai, Chofu, Kure and Onna), VOR Tx stations (black dots) and the Es propagation zone centered at Chofu, Tokyo. All the possible reflection points (i.e., mid-points between the Tx and $\mathrm{Rx}$ stations) are shown by the colored crosses (purple $=$ Sarobetsu, light blue $=$ Oarai, green $=$ Chofu, blue $=$ Sugadaira, orange $=$ Kure , and red = Onna). The locations of the four ionosonde stations in Japan are also shown.

$$
\begin{gathered}
\mathrm{ROT}=(\mathrm{TEC}(t)-\mathrm{TEC}(t-\delta t)) / \delta t \quad[\mathrm{TECU} / \mathrm{min}], \\
\mathrm{ROTI}=\sqrt{ }\left(\left\langle\mathrm{ROT}^{2}\right\rangle-\langle\mathrm{ROT}\rangle^{2}\right) \quad[\mathrm{TECU} / \mathrm{min}] .
\end{gathered}
$$

Note that here we use a 5 min time window for calculating the standard deviation of ROT. By taking the standard deviation of the fluctuating component of TEC, we can detect TEC disturbances in any 5 min time intervals, i.e., the use of ROTI would make it easier to extract Es in an automated fashion.

In this study we also employ data from four ionosonde stations in Japan, which are located in Wakkanai $\left(45.16^{\circ} \mathrm{N}\right.$, $\left.141.75^{\circ} \mathrm{E}\right)$, Kokubunji $\left(35.71^{\circ} \mathrm{N}, 139.49^{\circ} \mathrm{E}\right)$, Yamagawa $\left(31.20^{\circ} \mathrm{N}, 130.62^{\circ} \mathrm{E}\right)$, and Okinawa $\left(26.68^{\circ} \mathrm{N}, 128.15^{\circ} \mathrm{E}\right)$. The locations of these ionosonde stations are plotted in Figure 1. The temporal resolution of these ionosonde observations was 15 min during the interval of interest (i.e., ionograms were obtained every $15 \mathrm{~min}$ interval).

\section{Results}

Now we show how the 2D structure of Es can be visualized using EsAP and ROTI data by introducing an Es event on July 4, 2019, which is one of the most prominent Es cases in Japan during the summer months of 2019. Figure 2a shows the data from the four ionosonde stations in Japan. The color scale 

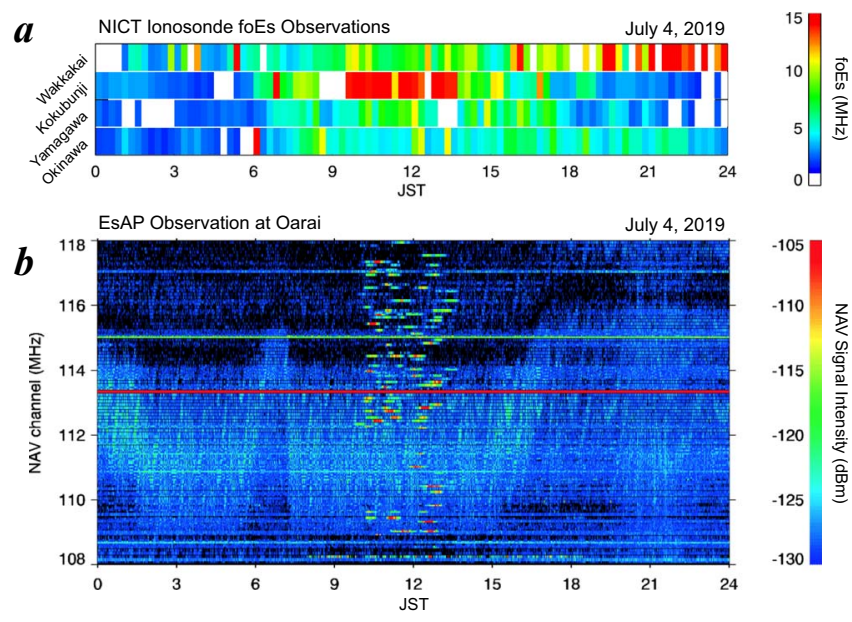

Fig. 2. Summary of a sporadic E event on July 4, 2019. (a) Timeseries data of the critical frequency of Es layer (foEs) obtained at four ionosondes in Japan. The vertical axis represents the location of ionosondes (Wakkanai, Kokubunji, Yamagawa, and Okinawa) and the color represents the critical frequency in MHz. (b) Anomalous propagation data in NAV channels in a format of spectrogram obtained at the Rx station in Oarai, Japan.

indicates the magnitude of the critical frequencies of Es trace (foEs) that can be used as a proxy for the intensity of Es. The foEs value from the Kokubunji station in Tokyo was mostly higher than $15 \mathrm{MHz}$ during $\sim 5 \mathrm{~h}$ from 0900 to 1400 JST (Japan Standard Time) indicating an appearance of Es at least above the Tokyo area during the daytime.

Figure $2 \mathrm{~b}$ plots the EsAP observation at the Oarai station near Tokyo. The vertical axis shows the frequency of NAV channels and the color scale indicates the received signal intensities. The frequency at which a continuous strong signal is received (reddish line in the middle) is due to direct wave propagation. In contrast to such a continuous signal reception, short duration enhancements of signal intensity are seen in many channels during the daytime, say from 1000 to 1400 JST, which are manifestations of EsAP. On this day, the receiver in Oarai detected the signature of EsAP in 49 channels when the ionosonde at Kokubunji observed an increase of foEs. Note that the possible location of Es causing the anomalous propagation should have been located in the western part of Japan, which is distant from the viewing area of the Kokubunji ionosonde. Later, we will discuss why we obtained a good correspondence between the ionosonde and EsAP observations at different locations by showing the ROTI maps.

Figure 3a shows a map of ROTI at 1150 JST on July 4, 2019 , when signatures of strong Es were seen in both the ionosonde and EsAP observations. Here, we used GPS-TEC data from representative 200 stations, out of the entire GPS stations of GEONET (GPS Earth Observation Network System) in Japan. From these 200 stations, we can retrieve data in near real time, which will be helpful for routine monitoring of Es in the future. The data have been mapped onto the geographic coordinate system by assuming the ionospheric pierce points (IPPs) at $100 \mathrm{~km}$, which is the general altitude of Es. When making the ROTI map, we have used several different IPP assumptions, for example at $300 \mathrm{~km}$ (i.e., F region). In that case, however, the shape of Es was much more blurred. The spatial structure was most discrete when we used the IPP at $100 \mathrm{~km}$ altitude. This fact strongly suggests that the derived ROTI map represents a structure existing exactly at the E region altitude, that is Es. The six colored diamonds mark the locations of the six $\mathrm{Rx}$ stations of EsAP observation. There is a region of increased ROTI in the middle of Japan islands, elongating more in the longitudinal direction, which is the possible manifestation of Es in the ROTI data.

Here, we map the structure of Es by combining the EsAP and ROTI observations. At each Rx station, we check the occurrence of EsAP for all the 193 channels every 5 min. For such extracted EsAP channels, the received frequencies are used to identify the possible Tx stations. Then, we simply map the mid-points between the Tx and Rx stations as a location of Es reflection. By mapping all of such intermediate reflection points on a map, we can visualize the $2 \mathrm{D}$ distribution of Es. However, the NAV system allocates the same frequency for multiple Tx stations because of the limited number of channels in the band. This produces multiple candidate points of Es even for a single EsAP event. Thus, only by using the frequency information, it is still difficult to pinpoint the correct source Tx station giving "real" location of Es.

The red crosses in Figure $3 \mathrm{~b}$ indicate all the candidate points (i.e., mid-points between all the Tx and Rx stations) of EsAP at 1150 JST on July 4, 2019. At this stage, the points are scattered in a wide area which does not allow us to identify the true spatial structure of Es. To overcome this problem, we applied an additional screening process to eliminate imaginary (ghost) points. Based on previous studies of Es (e.g., Maeda \& Heki, 2015) we assume that Es during daytime is localized in space (Es during nighttime is dominated by NW-SE elongating multiple structures though); thus, the true reflection points should be concentrated in a narrow region. Then, we calculated the number of EsAP channels detected in a 5 min interval, and extracted the true mid-points as points where more than $70 \%$ of the number of mid-reflection points exist within a box of $2^{\circ}$ in latitude $\times 3^{\circ}$ in longitude.

Figure $3 \mathrm{c}$ shows how this additional screening can reduce the imaginary points. Now all the imaginary (ghost) points have been screened out and the red crosses are concentrated in a narrow region. The cluster of red crosses is smoothly connected to the signature of Es in the ROTI data. Namely, both EsAP, ROTI and ionosonde (at Kokubunji, Tokyo) data observe the same Es structure extending in the longitudinal direction. Here, however, we should keep in mind that the current Es extraction method from the EsAP observation is only able to pick up a dominant (i.e., most prominent) Es frontal structure. At the same time, the method automatically discards the second most prominent Es structure. This is one of the limitations of the current algorithm for picking up true Es reflection from the EsAP measurement. In the future, we will update this screening process to be able to extract multiple Es traces appearing simultaneously.

Figure 4 demonstrates how the dynamical characteristics of Es can be imaged by the current approach. The six panels respectively show the combined data every $10 \mathrm{~min}$ interval from 1145 to 1235 UT on July 4, 2019. The red crosses, which are the reflection points of EsAP, move northward in tandem with the excursion of Es seen in the ROTI data. Based on the motion of EsAP reflection points, the average speed of the northward 

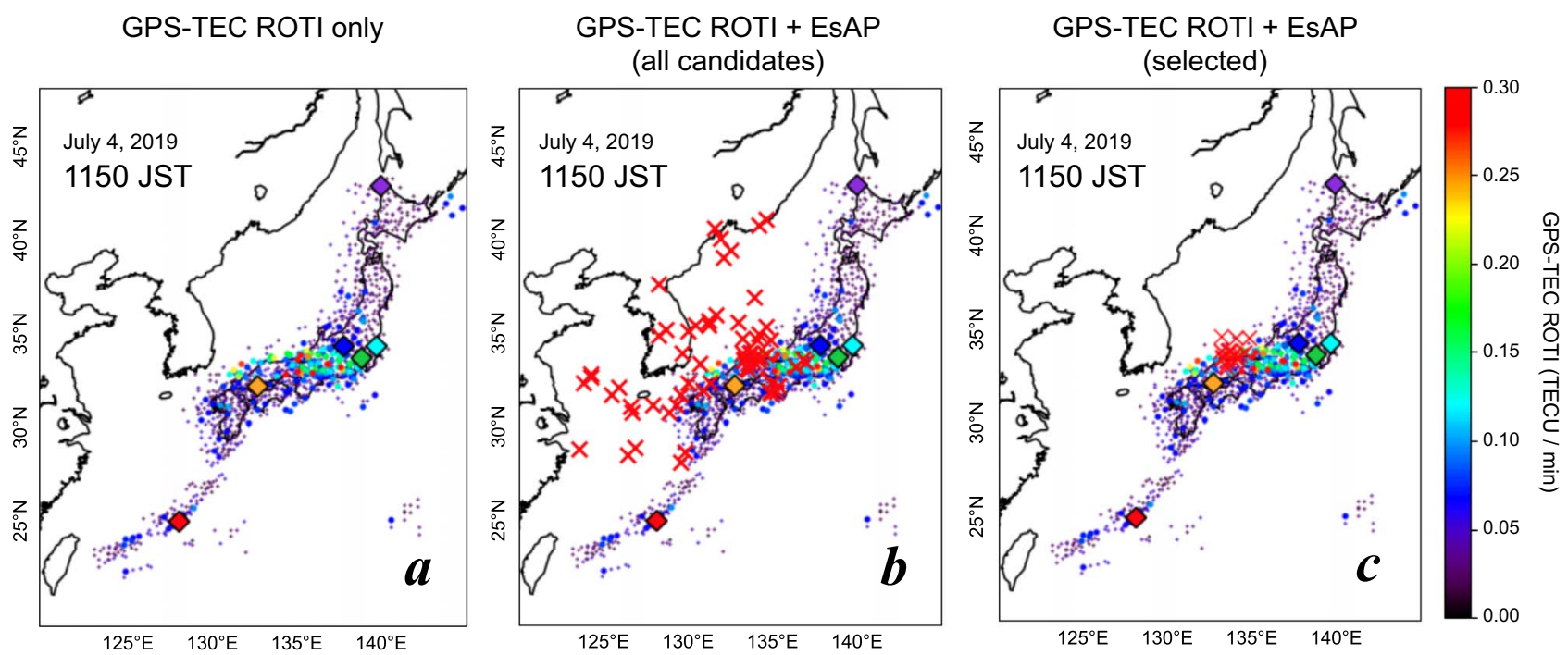

Fig. 3. (a) Map of ROTI at 1150 JST on July 4, 2019, which have been mapped onto an altitude of $100 \mathrm{~km}$. The diamonds show the locations of the six Rx stations of NAV signals (purple = Sarobetsu, light blue = Oarai, green $=$ Chofu, blue $=$ Sugadaira, orange $=$ Kure, and red = Onna). (b) Distribution of all the possible reflection points of EsAPs detected at the six Rx stations. The red cross marks represent the reflection points, which have been superimposed on ROTI map. (c) Same data as (b), but imaginary reflection points are filtered out.

excursion was about $63 \mathrm{~m} \mathrm{~s}^{-1}$ in this interval. The spatial extent of the Es structure mainly visualized by ROTI is roughly $110 \mathrm{~km}$ in the north-south direction and $730 \mathrm{~km}$ in the east-west direction.

\section{Discussion}

In this study, we introduced a method to visualize the twodimensional structure of the sporadic $\mathrm{E}$ layer by using the distribution of EsAP reflection points in combination with ROTI data. The results presented in Figures 3 and 4 demonstrate that the observed Es had a structure elongating more in the eastwest direction. In the previous section, we showed a good agreement between the ionosonde data at Kokubunji and EsAP observation at Oarai whose sensing areas had a large displacement. This discrepancy can be explained by the east-west elongating structure of the current Es event as visualized in Figures 3 and 4. That is, the east-west elongating Es passed through the sensing areas of ionosonde and EsAP observations at the same time, which caused almost simultaneous detection of Es by both the techniques.

Figure 4 demonstrated that the Es structure moved northward (i.e., in the direction perpendicular to its elongation) with a speed of about $60 \mathrm{~m} \mathrm{~s}^{-1}$. Maeda \& Heki (2015) showed that Es over Japan often shows a thin structure in latitude, but is more elongating in the longitudinal direction. In addition, during the event they analyzed, Es gradually moved northward while retaining its east-west elongation. These characteristics are consistent with the current observation, implying that we succeeded in tracking the 2D structure of Es with the current approach. In addition, good coincidence between the Es structures detected by the two methods proves that we are able to map the large-scale shape of Es by employing ROTI as a proxy for the existence of small-scale irregularities within Es. The GPS-TEC mapping of Maeda \& Heki (2015) is based on the precise estimation of residual component from the background whereas ROTI is the simple standard deviation of the variability component of TEC. This will make it easier to map the Es structure in an automated fashion, which is one of the advantages of the use of ROTI rather than the residual of GPS-TEC.

Direct comparison between the ROTI maps in Figures 3 and 4 with the residual GPS-TEC maps of Maeda \& Heki $(2014,2015)$ implies that the scale size of Es in the direction perpendicular to its elongation is much larger in the ROTI data than that in the residual GPS-TEC map. Namely, in the residual GPS-TEC maps of Maeda \& Heki $(2014,2015)$, frontal structures of Es tend to be much slimmer than those in the ROTI data. The method of Maeda \& Heki $(2014,2015)$ picks up pulse-like increases in the slant TEC time-series as crosssections of strong Es front. Since such a spiky variation mostly continues only for a few min (e.g., Maeda \& Heki, 2014; Sun et al., 2020), the current mapping method using ROTI might not be able to detect the sharp TEC pulses due to the relatively low temporal resolution $(5 \mathrm{~min})$. This may mean that the width of frontal Es structures can be overestimated in the ROTI map. More detailed comparison with the residual GPS-TEC method of Maeda \& Heki (2014) is needed to evaluate the limitation of the ROTI method.

Maeda \& Heki (2015) demonstrated that foEs needs to be extremely high (for example, higher than $16 \mathrm{MHz}$ ) to cause detectable disturbances in the slant TEC time-series; thus, the detection of Es using the residual of GPS-TEC time-series is feasible only for intense Es events. Similarly, the signature of EsAP tends to be detected only when foEs is higher than $15 \mathrm{MHz}$. Sakai et al. (2020) reported a case in which one of the aeronautical signals (ILS localizer) from an airport in Taiwan was received in Hiroshima, Japan, during an intense Es event. In that case, foEs, as estimated from the frequency of the aeronautical navigation signal and the possible geometry of the ray-path, was $19 \mathrm{MHz}$. That is, EsAP can also be used for mapping relatively intense Es events. 

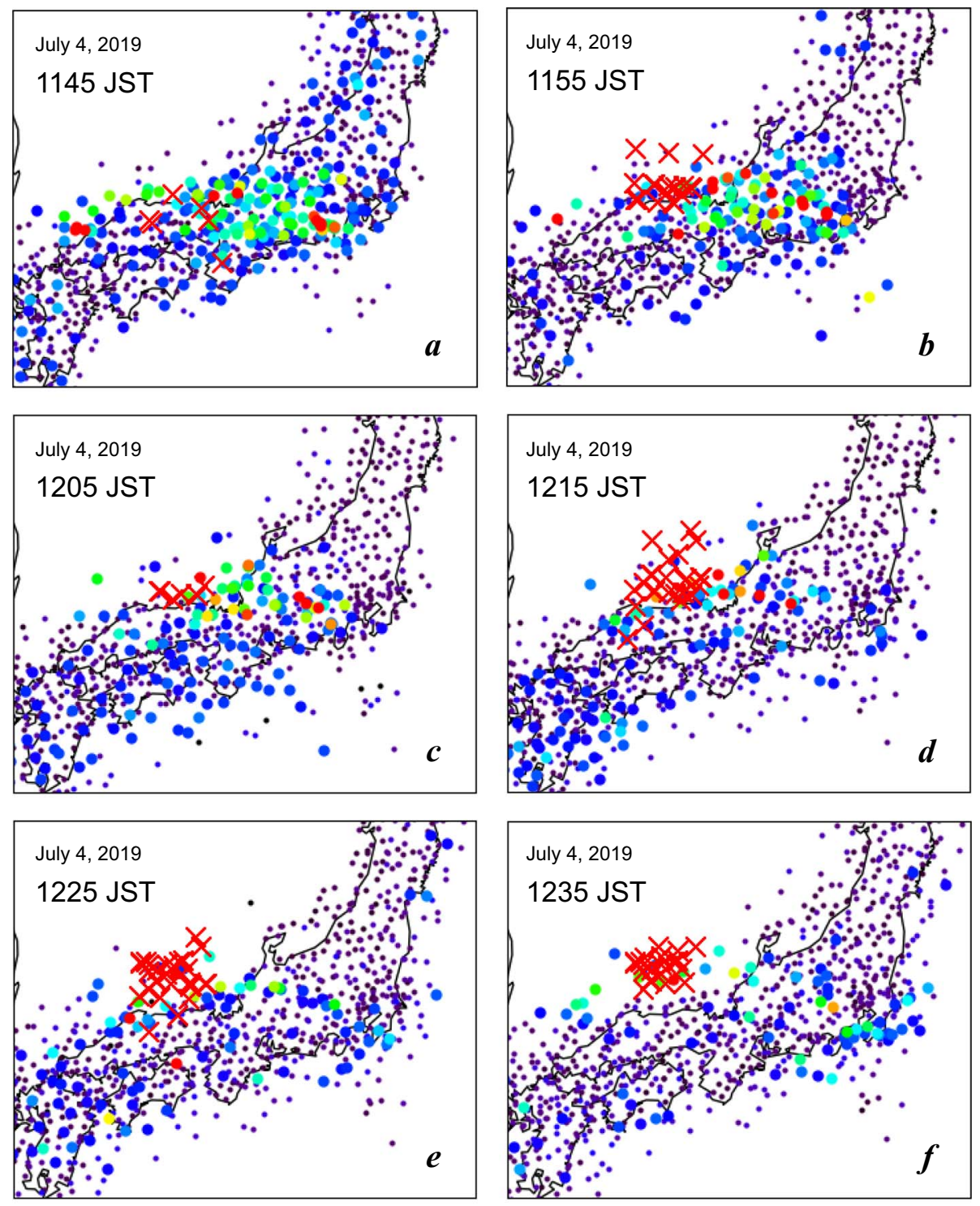

GPS-TEC ROTI (TECU / min)

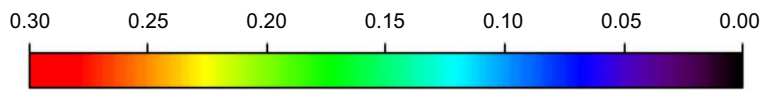

Fig. 4. Zoom-in views of the anomalous propagation data and ROTI sampled every 10 min from 1145 to 1235 JST on July 4 , 2019.

The ROTI is sensitive to the electron density variations not only associated with Es but also other ionospheric phenomena in the $\mathrm{F}$ region such as medium-scale traveling ionospheric disturbances (MSTID: Hunsucker, 1982). Hence, it is very difficult to detect Es when other ionospheric phenomena appear in the $\mathrm{F}$ region at the same time because thef density variation in the $\mathrm{F}$ region is generally larger than that in the E region. Even in such a situation, however, the mapping of Es with the EsAP observations is valid since the anomalous propagation of the VHF signals is not affected by other electron density perturbations in the $\mathrm{F}$ region.

As described above, the use of ROTI, instead of GPS-TEC itself, enables us to visualize Es in an automated fashion. Not only that, this technique for observing Es is expected to clarify the formation mechanism of electron density irregularities embedded within Es because ROTI is sensitive to the inhomogeneity in the spatial structure of Es. However, coverage of ROTI mapping over the sea is limited because the ground-based GNSS receivers are available only on land. In contrast, as shown in Figure 4, the mapping of EsAP reflection allows us to track the motion of Es outside the coverage of ROTI, especially in the sea area. In this sense, combination of EsAP and GPS-TEC observations is essential to visualize the structure of Es in a wide area including the sea area.

It has been suggested that electron density irregularities within Es can be a source of MSTID during summer nighttime (Cosgrove, 2007; Otsuka et al., 2007; Saito et al., 2007). To test this hypothesis, it is necessary to observe and compare the 
spatial structures of these two phenomena at different altitudes. GPS-TEC mapping is one of the ways to visualize the 2D structure of Es and MSTID. It is, however, difficult to detect them at the same time because the density perturbation associated with MSTID at the F region altitude is much larger than that of Es in the $\mathrm{E}$ region. On the other hand, detection of Es with the EsAP observation is not affected by any other phenomena in the F region; thus, it is possible to measure and compare the spatial structure of Es with that of MSTID imaged by the normal GPS-TEC mapping (e.g., Otsuka et al., 2013). Such a combination of EsAP and GPS-TEC observations will contribute to the detailed understanding of the generation mechanism of nighttime MSTID.

\section{Conclusions}

In this study, we introduced a method for mapping the twodimensional structure of sporadic $\mathrm{E}$ in a wide area by combining anomalous propagation observations of aeronautical navigation signals with ROTI data. To demonstrate the feasibility of this combined approach, we presented an event of Es in Japan on July 4, 2019, which was visualized by the current method. The mapping results indicate that the sporadic E showed an east-west extension with a spatial scale of approximately $730 \mathrm{~km}$ in east-west and $110 \mathrm{~km}$ in north-south directions, respectively. Sequential mapping images demonstrate that the entire Es structure was moving northward with a speed of approximately $63 \mathrm{~m} \mathrm{~s}^{-1}$. These spatial and dynamical characteristics are generally consistent with the previous Es mapping method of Maeda \& Heki (2015).

The current results indicate that the combination of ROTI with VHF anomalous propagation data from six receiving stations in Japan enables us to visualize the two-dimensional structure of Es over a wide area including the sea area with a time resolution of at least $5 \mathrm{~min}$. Since the VHF radio observation is not directly affected by the $\mathrm{F}$ region phenomena such as MSTID; thus, the anomalous propagation data can be used to identify the structure of sporadic E even when the sporadic E and MSTID occur simultaneously during nighttime. In particular, this method could be used for investigating the coupling between the $\mathrm{E}$ and $\mathrm{F}$ regions in the generation of MSTID.

Acknowledgements. KH thanks the staff of A. Nadai and M. Oshiro at the Okinawa Electromagnetic Technology Center of National Institute of Information and Communications Technology for supporting the VHF radio observation at Onna, Okinawa. KH also thanks A. Yamamoto at Japan Coast Guard Academy for supporting the VHF radio observation at Kure, Hiroshima. KH also thanks T. Yamahata for his efforts of developing the on-site observation software. $\mathrm{KH}$ is supported by JSPS-Kakenhi (18H04437). This study is also supported by a research grant from The Murata Science Foundation. A part of this work was supported by JSPS KAKENHI Grant Number JP15H05813, "The Project for Solar-Terrestrial Environment Prediction (PSTEP)". All the EsAP data used in this study are publicaly available at http:// gwave.cei.uec.ac.jp/ vor/data/. All the GPS-TEC ROTI data are available at https://www.enri.go.jp/cnspub/susaito/rocket/ data/20190704/20190704025000.tgz. The editor thanks Haris Haralambous and an anonymous reviewer for their assistance in evaluating this paper.

\section{References}

Cosgrove RB. 2007. Generation of mesoscale F layer structure and electric fields by the combined Perkins and Es layer instabilities, in simulations. Ann Geophys 25: 1579-1601. https://doi.org/10.5194/ angeo-25-1579-2007.

Davis RM, Smith EK, Ellyett CD. 1959. Sporadic E at VHF in the USA. Proc IRE 9: 762-769. https://doi.org/10.1109/JRPROC. 1959.287271.

Furuya M, Suzuki T, Maeda J, Heki K. 2017. Midlatitude sporadic-E episodes viewed by L-band split-spectrum InSAR. Earth Planets Space 69: 175. https://doi.org/10.1186/s40623-017-0764-6.

Haldoupis C. 2011. A tutorial review on sporadic E layers. In: Aeronomy of the Earth's atmosphere and ionosphere, Abdu MA, Pancheva D, Bhattaxharyya A (Eds.), Springer-Verlag, Berlin, pp. 381-394.

Hosokawa K, Sakai J, Tomizawa I, Saito S, Tsugawa T, Nishioka M, Ishii M. 2020. A monitoring network for anomalous propagation of aeronautical VHF radio waves due to sporadic $\mathrm{E}$ in Japan. Earth Planets Space 72: 88. https://doi.org/10.1186/s40623020-01216-Z.

Hunsucker RD. 1982. Atmospheric gravity waves generated in the high latitude ionosphere: a review. Rev Geophys 20: 293-315. https://doi.org/10.1029/RG020i002p00293.

Hysell DL, Burcham JD. 2000. The 30-MHz radar interferometer studies of midlatitude E region irregularities. J Geophys Res Space Phys 105(A6): 12797-12812. https://doi.org/10.1029/ 1999JA000411.

Maeda J, Heki K. 2014. Two-dimensional observations of midlatitude sporadic E irregularities with a dense GPS array in Japan. Radio Sci 49: 28-35. https://doi.org/10.1002/2013RS005295.

Maeda J, Heki K. 2015. Morphology and dynamics of daytime midlatitude sporadic-E patches revealed by GPS total electron content observations in Japan. Earth, Planets and Space 67: 89. https://doi. org/10.11186/s40623-015-0257-4.

Maeda J, Suzuki T, Furuya M, Heki K. 2016. Imaging the midlatitude sporadic E plasma patches with a coordinated observation of spaceborne InSAR and GPS total electron content. Geophys Res Lett 43: 1419-1425. https://doi.org/10.1002/ 2015GL067585.

Mathews JD. 1998. Sporadic E: Current views and recent progress. J Atmos Terr Phys 60: 413-435. https://doi.org/10.1016/S13646826(97)00043-6.

Otsuka Y, Onoma F, Shiokawa K, Ogawa T, Yamamoto M, Fukao S. 2007. Simultaneous observations of nighttime medium-scale traveling ionospheric disturbances and E-region field-aligned irregularities at midlatitude. J Geophys Res 112: A06317. https://doi.org/10.1029/2005JA011548.

Otsuka Y, Suzuki K, Nakagawa S, Nishioka M, Shiokawa K, Tsugawa T. 2013. GPS observations of medium-scale traveling ionospheric disturbances over Europe. Ann Geophys 31(2): 163-172. https://doi.org/10.5194/angeo-31-163-2013.

Pi X, Mannucci AJ, Lindqwister UJ, Ho CM. 1997. Monitoring of global ionospheric irregularities using the worldwide GPS network. Geophys Res Lett 24(18): 2283-2286. https://doi.org/ 10.1029/97GL02273. 
Saito S, Yamamoto M, Hashiguchi H, Maegawa A. 2006. Observation of three-dimensional structures of the quasi-periodic echoes associated with mid-latitude sporadic-E layers by the MU radar ultra-multi-channel system. Geophys Res Lett 33: L14109. https://doi.org/10.1029/2005GL025526.

Saito S, Yamamoto M, Hashiguchi H, Maegawa A, Saito A. 2007. Observational evidence of coupling between quasi-periodic echoes and medium scale traveling ionospheric disturbances. Ann Geophys 25(10): 2185-2194. https://doi.org/10.5194/angeo-25-2185-2007.

Sakai J, Hosokawa K, Tomizawa I, Saito S. 2019. A statistical study of anomalous VHF propagation due to the sporadic-E layer in the air-navigation band. Radio Sci 54: 426-439. https://doi.org/ 10.1029/2018RS006781.

Sakai J, Saito S, Hosokawa K, Tomizawa I. 2020. Anomalous propagation of radio waves from distant ILS localizers due to ionospheric sporadic-E. Space Weather 18: e2020SW002517. https://doi.org/10.1029/2020SW002517.

Shinagawa H, Miyoshi Y, Jin H, Fujiwara H. 2017. Global distribution of neutral wind shear associated with sporadic E layers derived from GAIA. J Geophys Res Space Phys 122: 4450 4465. https://doi.org/10.1002/2016JA023778.

Sun W, Wu B, Wu Z, Hu L, Zhao X, et al. 2020. IONISE: An ionospheric observational network for irregularity and scintillation in East and Southeast Asia. J Geophys Res Space Phys 125: e2020JA028055. https://doi.org/10.1029/2020JA028055.

Whitehead JD. 1989. Recent work on mid-latitude and equatorial sporadic-E. J Atmos Sol-Terr Phys 51: 401-424. https://doi.org/ 10.1016/0021-9169(89)90122-0.

Cite this article as: Hosokawa K, Kimura K, Sakai J, Saito S, Tomizawa I, et al. 2021. Visualizing sporadic E using aeronautical navigation signals at VHF frequencies. J. Space Weather Space Clim. 11, 6. https://doi.org/10.1051/swsc/2020075. 